\title{
Maritime Engineering: Referees 2020
}

The following is a list of referees who have reviewed papers for Maritime Engineering between 1 January 2020 and 31 December 2020. The Institution of Civil Engineers is very grateful for their assistance.

We are continually looking for suitable reviewers for papers submitted to Maritime Engineering. Papers published in the Proceedings of the ICE must be submitted to at least two independent referees to judge accuracy, style, impact, importance and interest.

If you are interested in reviewing articles on any topic related to maritime engineering, please submit your name, qualifications or $\mathrm{CV}$,

\section{Bianca Ãvila}

Juan Carlos Alcérreca-Huerta

Athanasios Angeloudis

Timothy Beckett

Denis Camilleri

Nicholas Christakis

Daniel Clemente

Lee Cunningham

Alan Cuthbertson

Tianjiao Dai

Zeki Demirbilek

Aggelos Dimakopoulos

Luke Farrier

Tiago Ferradosa
Richard Fletcher
Jassiel Hernández-Fontes
Chao Hou
Peng Hu
Tim Hunt
Paramban Jaipal
Doug Jenkins
Guomin Ji
Yonggang Jia
Luqi Ke
Xin Liu
Mario López

and areas of expertise. We are in need of individuals who will agree to review papers in a timely fashion (within 3 to 4 weeks of receipt) and provide confidential feedback to the Editorial Advisory Panel concerning the quality of the paper and any suggested revisions that would be appropriate.

If you are such a person, please contact Craig Schaper (tel.: +44 (0)207 665 2240; e-mail: craig.schaper@ice.org.uk) for more information on the referee process.

MIn Luo

Min Luo

Oleg Makarynskyy

Paulo Mendes

Edgar Mendoza

Guilherme Moura Paredes

António Mourão

Anna Mujal Colilles

Leticia Hereza Navarro

Peter Neville-jones

Pantelis Nikolakopoulos

Cliff Ohl

Jing-Jing Pan
Ashwin Parambath

Hui Qu

Subba Rao

Achilleas Samaras

Terence Stewart

Richard Swift

Amrit Verma

Robert Walker

Rongquan Wang

Peng Wu

Shun-Peng Zhu

Hai Zhu

Siyuan Zou 